\title{
L'enfant cueilleur des mots ou l'aventure pédagogique de Combemuger (1900-1908)
}

Hervé Terral

\section{(2) OpenEdition}

1 Journals

Édition électronique

URL : http://journals.openedition.org/trema/1526

DOI : 10.4000/trema.1526

ISSN : 2107-0997

Éditeur

Faculté d'Éducation de l'université de Montpellier

\section{Édition imprimée}

Date de publication : 1 septembre 2003

Pagination : 29-40

ISSN : 1167-315X

\section{Référence électronique}

Hervé Terral, «L'enfant cueilleur des mots ou l'aventure pédagogique de Combemuger (1900-1908)», Tréma [En ligne], 22 | 2003, mis en ligne le 13 octobre 2010, consulté le 08 mai 2019. URL : http:// journals.openedition.org/trema/1526; DOI : 10.4000/trema.1526

Ce document a été généré automatiquement le 8 mai 2019.

Trema 


\title{
L'enfant cueilleur des mots ou l'aventure pédagogique de Combemuger (1900-1908)
}

\author{
Hervé Terral
}

«Maintenant je vous demande de me perdre et de vous trouver»

F. NIETZSCHE (Ainsi parlait Zarathoustra) cité par A. Perbosc en exergue de «La

Tradicion occitana », in Occitania. 1905, $\mathrm{N}^{\circ} 7$.

1 Connaît-on aujourd'hui, dans la sphère pédagogique, Antoine Crépin (dit Antonin) Perbosc (1861-1944), instituteur laïque et chantre de l'occilanisme littéraire et culturel ? Les candidats au CAPES d'occitan / langue d'Oc sans doute puisqu'il fut à leur programme d'études en 2000 et 2001 ! Mais, au delà ? Il est, hélas, fort à parier que, si l'écrivain, célébré de son vivant à Barcelone (un recueil de contes traduits en catalan y est publié dès 1905) comme à Paris (Prix « Vignes de France » partagé avec Paul Fort, en 1932), n'est pas tout à fait oublié - ainsi qu'en témoignent des rééditions récentes de ses œuvres majeures, L'Aradaet Lo libre del Campèstre, le pédagogue Perbosc appartient désormais au royaume des ombres. La forme la plus avancée de ce que Durkheim nommait en 1905 le «misonéisme" (i.e. la «haine de la nouveauté») consiste, en effet, à ignorer, délibérément ou non, ce que fut l'innovation d'hier: on est parfois assuré alors, par cet artifice ou cet oubli, d'une carrière certifiée... d'« innovateur » institutionnel. Ainsi m'a-til été donné de constater que, dans un IUFM porté à l'exaltation de ses résultats nationaux aux concours, celui de Midi-Pyrénées pour le nommer, le fait qu'UN groupe de professeurs stagiaires des écoles d'une part, UN groupe de leurs formateurs d'autre part, pussent travailler... en groupe se voyait élevé vers 1998 au rang d'une nouveauté sans réserves, saluée par le Monde de l'Éducation lui-même. Mais il s'agissait là d'un autre siècle... Il est vrai que l'on pouvait acheter, au même moment et à fort bas prix, auprès des «puceux » du marché Saint-Sernin de Toulouse des ouvrages de R. Cousinet (tel un exemplaire de L'Éducation nouvelle, 1950, avec une fiche de lecture à l'intérieur datant de... 1996) issus de la Bibliothèque de feu l'école normale, portant le très officiel tampon: « Annulé »! 
2 Dans un tel contexte, rendons donc à Perbosc ce qui est à Perbosc en large part (tant l'on n'est jamais complètement sûr que personne ne vous a quelque peu précédé1): une singulière aventure pédagogique en porte témoignage - qui lui vaudra, par ailleurs, une reconnaissance pleine et entière d'ethnologue, comme l'attestent sa présence dans le Manuel de Folklore français (lre éd., 1943) de A. Van Gennep et, plus encore peut-être dans une perspective d'ensemble, la plaque apposée sur la Bibliothèque municipale de Montauban (Tarn-et-Garonne) dont il fut, au soir de sa vie, responsable :

«Fondator de l'occitanisme

Poèta e reformator de la lenga d'Oc

Pedagogue, etnografe, bibliotecari,

Majoral del Felibritge (Cigala de la Libertat) »

Oui, Perbosc fut tout cela à la fois - au risque de surprendre ! Et, si l'on peut s'intéresser plus particulièrement à tel ou tel aspect d'une œuvre immense qui reste encore à découvrir ${ }^{2}$, on ne saurait trop longtemps la dissocier en des champs dispersés sous peine d'en perdre la signification majeure : un combat culturel et politique pour « faire entrer la langue d'Oc à l'école » - dans un contexte où, quel que soit l'intérêt porté aux « petites patries » et autres "pays " ${ }^{3}$, un interdit majeur demeure: seul le français est langue d'enseignement dans les écoles primaires publiques (article 14 du Règlement modèle des écoles primaires, 7 juin 1880) et, cela va sans dire, au delà (enseignement secondaire destiné alors aux élites).

\section{Un lieu privilégié dans l'histoire de la pédagogie}

4 A la fin du XIX ${ }^{e}$ siècle, Comberouger est un petit village de Lomagne, où viennent se mêler les dialectes gascon et languedocien de l'occitan. Zone de confluence au profil bien traditionnel néanmoins : 500 âmes, des paysans surtout, des artisans et des commerçants aussi. Quand A. Perbosc en devient, en 1893, l'instituteur public des plus grands et le secrétaire de mairie (madame Perbosc devenant quant à elle l'institutrice des plus petits), il quitte ses chers côteaux du Quercy à la suite d'une obscure intervention du maire de Laguépie (où il était en poste) visant à faire obtenir l'emploi local à son... cousin : la République est ce qu'elle est - même si elle souhaite alors que ses instituteurs ne soient pas nommés là même où ils sont nés sous peine de perdre leur aura et leur crédit ${ }^{4}$ ! Heureuse intervention, somme toute, puisqu'elle va mettre le jeune maître, déjà Majorai du Félibrige à l'initiative de Mistral lui-même (Cigale de la Liberté, 1892) mais porté vers un Félibrige "rouge », fédéraliste, libertaire, en présence d'une Occitanie qu'il s'efforce de faire émerger de son lointain passé (celui des troubadours et des chartes fondant les bastides) et de retrouver dans la langue quotidienne du peuple ${ }^{5}$.

5 Sans trop d'hésitation, on peut dire que, si l'arrivée à Comberouger ne fut pas du tout désirée (Perbosc parle même dans sa correspondance de "trou perdu»), elle offrit très vite le terrain, voire le terreau, où ses convictions allaient, au sens littéral, s'enraciner : Comberouger présente, en effet, cette petite différence, ce petit écart, qui permettent, chacun le sait, de s'interroger sur soi... et les autres, de se construire une identité, déjà fortement affirmée à vrai dire. Non pas seulement celle de la "petite patrie » chère aux maîtres-penseurs de la pédagogie officielle (E. Lavisse en premier lieu), Heimat à la française, quoi qu'il en soit... ouvrant sur «la plus grande France » (métropole et toutes colonies confondues dans un seul ensemble émancipateur de l'Humanité tout entière), mais aussi celle de la «grande patrie» qui, pour Perbosc va prendre le nom 
$d^{\prime}$ «Occitania » ${ }^{6}$. La toile de fond est là, obsédante, et s'impose néanmoins presque «naturellement» dans les travaux et les jours d'une communauté, dans les rires et les jeux des enfants, dans ce que les pédagogues de "l'Éducation Nouvelle» vont au même moment dénommer, de façon générique, la « vie ». Perbosc participe à sa façon, c'est-àdire avec indépendance, de ce vaste mouvement rénovateur de la pédagogie $-\mathrm{d}$ 'où vont sortir les Cousinet et les Freinet dans l'entre-deux-guerres.

Elève extrêmement brillant de l'école normale de Montauban (où il fût admis avec un 20 en composition française !), Perbosc, fils de petits métayers quercynois, est un « hussard noir de la sévérité ", un "nourrisson de la République», selon les fortes acceptions de Péguy dans L'Argent (1913) 7 . À ce titre, il a fait siennes les recommandations de ses mentors : la "pédagogie intuitive » défendue par F. Buisson (1878), dans le droit fil des Seminarien germaniques, et la "méthode active ", prônée en Sorbonne par le titulaire de la nouvelle chaire de Science de l'Éducation (au singulier alors) H. Marion (1888) doctrines relayées par la presse pédagogique nationale (Revue pédagogique; Manuel général) et départementale (Bulletin de l'Instruction primaire) : l'une et l'autre font la part belle à "l'observation », matrice de la célèbre « leçon de choses » in situ, voire à ce qui prendra nom aux lendemains de la Libération d'« étude du milieu ». Le maitre est, par exemple, invité à faire collectionner par les élèves roches, minéraux et plantes diverses; plus encore, à construire de véritables petits musées scolaires qui, au demeurant, peuvent lui apporter une certaine notoriété à l'occasion d'expositions officielles ou de concours scolaires... De même est-il sollicité pour développer l'histoire locale: la monographie communale devient un genre quasi obligé (elle sera même dans les années 20 élevée à la dignité de «mémoire professionnel » dans le cadre des études de sociologie des écoles normales) - Perbosc y gagnera même un prix pour celle de Lacapelle-Livron (1886). Une circulaire du 25 février 1911 sur les vertus heuristiques de cette « histoire locale ", due à un éphémère ministre de l'Instruction publique originaire de la Drôme, le félibre Maurice Faure, autorisera un temps quelques espoirs, chez les régionalistes divers, de voir leur cause enfin prise en considération par la République « une et indivisible » (ante 1914-1918, faut-il préciser!). D'autre influences intellectuelles se font sentir, plus sulfureuses, au premier rang desquelles il importe de mentionner celle du comte Léon Tolstoï, grand critique des manuels scolaires et promoteur du texte libre: ses œuvres, relatant son travail libertaire d'esprit à l'école de Iasnaïa Poliana, créée par ses soins pour ses petits moudjiks, sont traduites en français dès les années 1890, telle La liberté dans l'école, introduite en France par M. Bréal qui voit dans l'auteur un «noble et profond penseur » - lors même que G. Compayré, autorité pédagogique de la période, parle d'un «Rousseau slave [...] au fond troublé et bizarre " (Revue pédagogique, 1890, № 6, pp. 544-552). On peut assez légitimement penser que Perbosc eut connaissance de ces débats et fit son choix...

Dès 1886, Perbosc s'est prononcé dans la Tribune des Instituteurs et Institutrices de France ${ }^{8}$ pour l'utilisation des « patois » en classe, redécouvrant en cela les remarques du linguiste M. Bréal (Quelques mots sur l'Instruction publique en France, 1872); il se pique même de donner des devoirs « en patois " à ses élèves, il est rappelé à l'ordre par l'Inspecteur J. Pouillot (1890) mais... il accède, au même moment, au Bulletin de l'Instruction Primaire du Tarn-et-Garonne avec un "Toast au Quercy et à ses poètes" en langue d'oc... Parallèlement, son ami et alter ego Prosper Estieu (1860-1939), objet de déplacements administratifs quasi annuels dans son département, l'Aude, se voit, lui aussi, sanctionné pour ses plaidoyers en faveur de l'occitan dans les conférences pédagogiques 
d'instituteurs et la revue Mont-Segur (1902) qu'ils co-animent de 1896 à 1904. Un mouvement semblable existe, remarquons-le, en Béarn et en Gascogne avec l'instituteur Sylvain Lacoste (1862-1930) ou en Provence avec le chanoine Jean Aurouze ${ }^{9}$, disciple du Frère Savinien (J. Lhermite). L'instituteur béarnais Jean-Victor Lalanne (1849-1924) avait, quant à lui, déjà recueilli des contes, édités anonymement en 1890. L'érudit gersois JeanFrançois Bladé (1827-1900) avait de même précédé Perbosc en Lomagne et connu le succès de plusieurs éditions parisiennes pour ses contes et poésies populaires.

\section{Une expérience singulière}

C'est le moment que choisit Perbosc pour lancer une authentique expérience pédagogique, annonciatrice des travaux ultérieurs d'un Célestin Freinet : faire collecter par ses élèves contes et chansons dans le cadre d'études dites "folkloriques", les transcrire et les analyser... après la classe (pour ne pas être sanctionné par l'inspection). Située dans le prolongement des premiers travaux de type monographique, classique de l'institutorat, l'expérience de Comberouger peut, en effet, être considérée comme une réponse délibérée, à la fois scientifique, pédagogique mais aussi politique, au processus d'acculturation clairement dénoncé dès les années 1886-87. Précisons toutefois qu'elle ne s'inscrit pas chez Perbosc dans une posture tournée vers la glorification d'un passé peu ou prou angélique : l'homme s'affirme comme un novateur, porté par exemple sur la photographie (à laquelle il initie le curé du village), le phonographe et la bicyclette!

9 Un programme fort et résolu émerge alors, résumé plus tard en une phrase de sa correspondance avec Estieu (27 août 1911) : «Faire entrer la langue d'oc à l'école. » Pour l'heure (en 1900), il écrit à son ami :

«Si tu tiens à savoir ce que j'ai fait depuis octobre, apprends que j'ai folklorisé surtout. Et j'ai entrepris une cuvre originale que tu verras réalisée dans un an à peu près. En voici le plan:

Monographie d'un village:

"I. La Vie au village. 1. Autrefois 2. Aujourd'hui.

»II. Le Folklore.

"1. Contes populaires: Aventures merveilleuses; contes épiques. Contes mystiques et superstitions. Contes familiers. Récits.

"2. Poésies populaires: Romances. Chansons d'amour. Chansons de travail. Chants spéciaux. Chansons pour petits enfants. Chants historiques. Récitatifs, formules, etc.

"3. Traditions et légendes.

"4. Proverbes et locutions proverbiales.

"5. Devinettes populaires.

»6. Jeux populaires.

"L'originalité, c'est que je ne suis que le directeur de ce travail d'équipe et que ce sont mes élèves, une quinzaine de garçons et de filles de dix à treize ans qui le font.

" La première partie se déroulera sur une suite de petites monographies (100 environ) sur tous les sujets relatifs au terroir. Ce seront simplement des devoirs d'élèves bien coordonnés. "La deuxième partie est la plus avancée. On m'a recueilli jusqu'ici quatre-vingt contes, souvent informes, parmi lesquels (quelle surprise pour moi!)il y a des chefs-d'œuvre, certains inédits. Tout cela est transcrit et traduit patiemment, et le bloc augmente tous les jours (entre parenthèses, il y a jusqu'ici une quinzaine au moins de ces contes qui peuvent être publiés, et j'ai songé à la Revue des Pyrénées, à La tradition...) Pour les proverbes et les devinettes, le travail est à peu près fini : il est important. Pour les poésies populaires, ça commence; je ne compte pas trop sur ce filon; mais j'aurais peut-être les mêmes surprises que pour les contes. » [Je souligne] 
Le 14 mai 1900, Perbosc réitère : "Je suis en plein dans mon folklore... et je suis de plus en plus émerveillé des contes que mes élèves recueillent, écrivent, traduisent et transcrivent avec autant de frénésie que moi-même... C'est un travail formidable pour des enfants et un travail qu'un homme ne pourrait pas faire... Je fais noter ces contes en absolu patois de Comberouger. Il y a des morceaux bien plus scatologiques [que ceux cités habituellement].

$C^{\prime}$ est à peine si nos terriens s'en doutent. Ils n'ont pas, eux, nos hypocrites pudeurs! ${ }^{10}$ nouveau poste, ce sera de fonder une société traditionniste ${ }^{11}$. Celle que j'ai fondée ici, la première du monde, je suppose - fonctionne depuis janvier. Ses membres, au nombre de dix-huit, sont des enfants, garçons et filles, de huit à treize ans. Ce sont surtout les filles qui font d'excellentes folkloristes.» En 1906, Perbosc fera encore part de son enchantement par une belle formule : "Je suis enfantinement heureux en leur société."

11 Si le bilan proprement monographique (la $1^{\text {re }}$ partie annoncée) demeure maigre, la collecte des ethno-textes (aujourd'hui déposés à la Bibliothèque d'étude et du patrimoine de Toulouse) est plus que conséquente. L'innovation pédagogique aussi. Elle s'accompagne :

- de la création de la « Société traditionniste » dont les statuts sont déposés le 15 janvier 1900 et qui existera jusqu'au départ de Perbosc (en octobre 1908). L'article 5 de ces derniers précise : «La société fonctionne sous la direction de l'instituteur qui fait partie du bureau avec voix prépondérante. Le bureau comprend un président, un vice-président et un secrétaire. Ces trois derniers membres [des élèves] sont élus pour un an. $»^{12}$ Perbosc envisage de créer au même moment une « Fédération des sociétés traditionnistes scolaires du canton de Verdun-surGaronne » (13 septembre 1900) à l'occasion d'une fête patronnée par le fondateur de la Fédération régionaliste française, le professeur marseillais Jean Charles-Brun (1870-1946) : il s'agirait alors de "n'avoir pas l'air de faire une politique anticléricale tout en en faisant " (sic) ${ }^{13} \mathrm{II}$ faut affirmer, en effet, « Le Félibrige fédéraliste et libertaire » (12 septembre 1901 )... contre le Félibrige conservateur et clérical sans aucun doute.

- du début d'une correspondance scolaire avec les élèves des écoles d'Auvillar, distante d'une vingtaine de kilomètres (Tarn-et-Garonne).

De tels faits ne peuvent qu'inciter à rappeler l'avis de Célestin Freinet dans L'Éducateur à propos de la déclaration du Mouvement Laïque des Cultures Régionales ( Les Langues de France et l'Ecole Publique», numéro commun des "Cahiers Pédagogiques de l'Institut d'Etudes Occitanes » et de la revue bretonne «Ar Falz $\left({ }^{14}\right.$ :

"Il fut un temps, ou début du siècle, où l'École publique menait une lutte à mort contre les langues régionales 〈patois `. C'était le temps où les enfants n'avaient pas le droit de parier une langue maternelle, non seulement à l'École mais en récréation ou même dans la rue. L'invention du «signe〉 était le plus belle trouvaille de la scolastique d'alors. L'instituteur faisait un signe sur un sou. Il s'agissait pour le possesseur du 〈signe〉, de se débarrasser du sou en le donnant à un autre élève qu'il aurait surpris prononçant seulement un mot de patois.

" On imagine ce qu'a pu donner ce système perfectionné de délation. Heureusement la vie se rit toujours de la scolastique, les langues régionales ne sont pas mortes; elles refleurissent et leur enseignement officiel entre peu à peu dans la réalité de notre éducation nationale.

"Nous nous en réjouissons sans réserve, et cela par principe essentiel de nos techniques. "L'ancienne pédagogie n'avait rien à demander à l'enfant et son comportement était en somme conforme à ses propres principes. Et tant que ces principes demeurent, le comportement des maîtres ne saurait être changé. « Il n'est ni nécessaire ni même utile au maître qu'il connaisse la langue des enfants qu'il instruit, puisque jamais il n'aura besoin d'y recourir en classe. Il y a plus du point de vue pédagogique, il vaudrait même mieux qu'il ne la sût pas `. Y a-t-il lieu de tenir compte des quelques 
mots bretons péniblement acquis par les enfants dans la vie familiale et sociale? ‘ Je ne le crois pas, disait un Inspecteur d'Académie. Mieux vaut admettre que l'enfant ne soit rien, et commencer avec lui par le commencement, comme on fait à l'école maternelle. )

"C'est parce qu'on nous avait dit la nécessité au contraire de commencer par le commencement que nous intégrons les langues maternelles dans nos méthodes naturelles. "Il serait même souhaitable que nos journaux scolaires fassent une part plus grande aux textes d'enfants dans leur langue maternelle... »

13 Si Freinet ne s'est jamais (à ma connaissance) revendiqué explicitement de Perbosc, il ne pouvait pas, vraisemblablement, l'ignorer ${ }^{15}$ : quoi qu'il en soit sur ce point, la parenté objective des projets et des démarches saute aux yeux.

\section{Les enjeux politiques d'une innovation pédagogique}

14 Au delà de la simple collecte de contes, il convient d'appréhender combien ce travail n'est qu'une pièce dans l'activité multiple, voire dispersée, de l'auteur (animateur d'un cours d'adultes et secrétaire de mairie par ailleurs). Sa réflexion court, en effet, sur des considérations devenues très actuelles un siècle plus tard :

«Le Félibrige me parait tué aux trois-quarts. Mais le traditionnisme gagne peut-être, et les idées de décentralisation aussi. Et encore la chance de voir se former les États-unis d'Europe. Attention à ceci: alors refonte totale ou cataclysme. Emergeront peut-être, si des énergies le veulent, non pas la Provence, qui n'a que l'enthousiasme verbal, mais l'Aquitaine, le Languedoc, la Catalogne. C'est surtout en la Catalogne que j'ai foi. Autrefois, il fallait des monts pour séparer les races. L'avenir fera peut-être des Pyrénées, au contraire, l'épine dorsale d'un État fondé sur des bases nouvelles. Mais quel poids cette Espagne... Je n'achève pas l'ébauche de ce rêve. Que de choses à dire là dessus! Une réalité domine : la plupart des langues mourront. Laquelle survivra? Il semble impossible que ce soit la nôtre et pourtant je vois des raisons de croire que c'est une fille du latin qui tuera l'anglais, l'allemand, le russe. Et pourquoi cette survivante ne serait-elle pas notre langue?» (A. Estieu, 27 juin 1900).

Le propos est, à l'évidence, utopique. Il prolonge à sa façon les convictions premières de l'auteur : "Dans notre dernière conférence pédagogique, j'ai combattu - seul contre tous - l'idée de... haine. Mais, à part ça, l'heure du cosmopolitisme n'est pas venue... Nous sommes une nouvelle école dans le Félibrige : non plus des séparatistes, mais des cosmopolites - ce qui en somme revient à peu près au même. Le Campèstre - l'Humanité!» (A. Estieu, $1^{\mathrm{er}}$ juin 1892). Cette «Humanité », Perbosc la retrouvera encore, au soir de sa vie, quand il étudiera avec l'archiviste du département, Séverin Canal, les Coutumes médiévales de différentes communes du Quercy.

De la monographie à l'étude historique érudite en passant par l'étude du milieu, la voie ouverte par Perbosc est particulièrement féconde et l'expérience de Comberouger (1900-1908) sera saluée en son temps... par l'Inspecteur général Edouard Petit (dans L'école de demain (1901), il fait de ce travail sur les "petites patries » un modèle !). De même, émergent de cette œuvre complexe des Contes de la vallée du Lambon - complétés par les Contes de la vallée de la Bonnette, recueillis à l'identique par un collègue et ami de Perbosc, l'instituteur Hisnard, auprès de ses élèves de Loze (Tarn-et-Garonne) ${ }^{16}$.

\section{Conclusion}

Au soir de sa vie (1943), Perbosc confiera néanmoins son désenchantement au folkloriste André Varagnac: "Deux langues auraient pu vivre côte à côte, en gardant chacune sa 
personnalité et sa pureté, non seulement sans se nuire l'une à l'autre, mais en gagnant l'une et l'autre à être cultivées parallèlement. Au lieu de cela, le Français fait une guerre incessante aux parlers du peuple... » ${ }^{17} \mathrm{~L}$ 'administration scolaire n'a pas toujours eu, il est vrai, la largeur de vue de l'Inspecteur général Petit, belle exception dans la période, et, chacun le sait, l'exode rural a largement contribué, particulièrement dans les années 1950-60, à l'acculturation des populations occitanophones, promptes à trouver par ailleurs dans l'usage exclusif du français la marque tangible de leur ascension sociale...

Aujourd'hui, à l'occasion d'une journée d'études organisée par le Collège d'Occitanie et l'IUFM Midi-Pyrénées (le 8 décembre 2000), il nous fut donné de voir sur le tableau de l'école, quasi-inchangée, de Comberouger, devenue une petite commune-dortoir de la «troisième ceinture de l'agglomération toulousaine »... la trace écrite d'un cours d'anglais - dans le droit fil des recommandations du ministre Claude Allègre ${ }^{18}$ pour ne plus considérer cette nouvelle lingua franca comme « langue étrangère ». Ouvert à toutes les cultures, Perbosc s'en fut, vraisemblablement, réjoui, mais il se fut désolé, sans aucun doute, du sort fait à sa propre langue "reirala $»^{19}$. Pour autant, à l'heure de la Charte européenne des langues régionales et minoritaires, le combat d'un Perbosc ou d'un Estieu en terre occitane, d'un Yann Sohier en Bretagne quelques décennies plus tard, et de bien d'autres ailleurs, continue : pour la dignité de toutes les langues à tout le moins. En ce sens, leur démarche demeure, par delà tel ou tel aspect particulier prenant place dans l'histoire complexe des idées pédagogiques, profondément innovante parce qu'inscrite dans un héritage clairement revendiqué. Condition sine qua non, faut-il le rappeler...

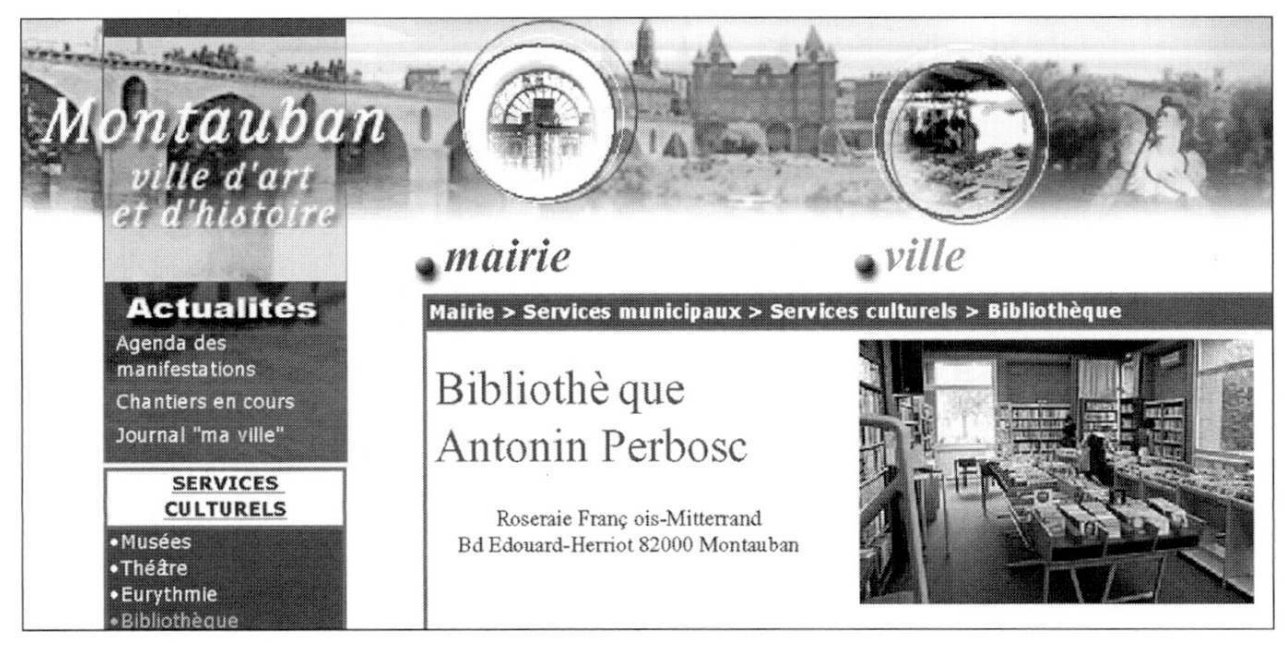

Page web de la bibliothèque municipale dont en son temps Antonin Perbosc fut le bibliothécaire. En voici l'URL : http ://www.ville-montauban.fr/rnairie/service-municip/cullture/biblio.

\section{BIBLIOGRAPHIE}

BOUTAN P. : La langue des messieurs. Paris, A. Colin, 1996.

BOUTAN P. : Michel Bréal, linguiste et pédagogue. Paris, Hatier, 1998. 
CHANET J.-F. : L'École républicaine et les petites patries. Paris, Aubier, 1996.

PERBOSC A. : Manifestes occitans. Montauban, Éd. Cocagne, 1974.

PERBOSC A. : Récits et Contes populaires de Gascogne - I (réunis par sa petite-fille S. CézeracPerbosc). Paris, Gallimard, 1979, avec le concours de G. Passerat et N. Sabatié.

TERRAL H. : Les savoirs du maitre. Enseigner de Guizot à Ferry. Paris, L'Harmattan, 1998.

TERRAL H : L'École de la République, une anthologie (1878-1940). Paris, Centre National de la documentation pédagogique, 1999.

THIESSE A.-M. : Ils apprenaient la France. Paris, Éd. de la Maison des sciences de l'homme, 1997.

\section{ANNEXES}

Le fi Ballet - Le fin Valet ${ }^{20}$ In Contes de la vallée du Lambon, Montauban, «Société des Etudes locales dans l'Enseignement public. Groupe de Tarn-et-Garonne », Paul Masson Libraireéditeur, 1914, pp. 51-56.

\section{Le fi Ballet}

I auè, un cop, un ome que louguèc un bailet. Le prumer jour, i dissec :

«Bailet, eau ana laura."

Atchi que parton, cadun ambe soun parelh e soun arai, sans res prengue per minja. Quand estec ouro de dina, le mèste dissec : « Tchi sab, bailet, se haziam coumo tchi dino ? - Iè ! mèste, dissec le bailet, dina atau nous emplenara gaire l'estoumac, mès harem coumo vèlhetz. »

\section{Le fin Valet}

II y avait, une fois, un homme qui loua un valet. Le premier jour, il lui dit :

"Valet, il faut aller labourer."

Voilà qu'ils partent, chacun avec sa paire de bøeufs et son araire, sans rien prendre pour dîner. Quand vint l'heure de dîner, le maitre dit :

Qui sait, valet, si nous faisions comme qui dîne?

- Hé! maître, dit le valet, dîner ainsi ne nous emplira guère l'estomac; mais nous ferons comme voudrez.»

Hasqueon dounc coumo tchi dino, apèi tournèon laura.Mès atchi que le mèste s'apercebec qu'en laurant le bailet passauo toutjour, toutjour dins la mèmo rego e i dissec : « È ! que hès, que hès, bailet ?- Iè ! mèste, vengui de hè coumo tchi dino ; aro, hèu coumo tchi lauro !» Le se, en anguent au lhèit, l'ome dissec à sa hemno :« Douman, le bailet anira houja la vinho : i balharas de que beue e minja. »L'endouman, dissec au bailet :« Bailet, te eau ana houja la vinho. Atchi as le houssoun e la biasso garnido.- Mèste, i vau. »

Ils firent donc comme qui dîne, puis ils se remirent à labourer. Mais voilà que le maître s'aperçut qu'en labourant le valet passait toujours, toujours dans le même sillon, et il lui dit: «Hé! que faistu, que fais-tu, valet?

- Hé! maître, je viens de faire comme qui dîne; maintenant, je fais comme qui laboure!» Le soir, en allant au lit, l'homme dit à sa femme:

- «Demain, le valet ira houer la vigne. Tu lui donneras de quoi boire et de quoi manger.» Le lendemain, il dit au valet : 
- « Valet, il te faut aller houer la vigne. Voilà ta houe et ta besace garnie.

- Maître, j'y vais. "

Atchi le bailet à la vinho : « Adiu, vinho, vinharasso.- Que i a dins ta coujarasso ?-

Machanto vinadasbo.- E dins toun sacas ?- Machant pan d'ordi.- Coucho-te. Jordi. »

Le se, quand le bailet arribèc à la bordo, le mèste i demandèc : » È be, bailet, n'as pla hèit ?

- 0 ! tioc, mèste, jusc' à la souqueto torto. "L'endouman, le mèste partie auant le hailet e vie que la vinho n'èro pas brico houjado. S'amaguèc débat la barralho, au moument ount le bailet arribao, e l'entendec que dizè :

Voilà le valet à la vigne:

"Adieu, vigne, vignerasse. - Qu'y a-t-il dans ta gourde? - Mauvaise piquette. - Et dans ton sac? Mauvais pain d'orge - Couche-toi. Georges."

Le soir, quand le valet fut de retour à la borde, le maître demanda : "Eh bien valet, en as-tu fait beaucoup?

- Oh! oui, maître, jusqu'à la souche torte. "

Le lendemain, le maître partit avant le valet, et vit que la vigne n'était pas houée du tout. Il se cacha sous la haie, au moment où le valet arrivait, et il l'entendit qui disait :

« Adiu, vinho, vinharasso. - Que i a dins ta coujarasso ? - Machanto vinadasso. - E dins toun sacas ? - Mâchant pan d'ordi. - Coucho-te, Jordi. »Le se, quand le bailet estec tournat à la bordo, le mèste i demandée : « È be, bailet, n'as pla hèit ?- Autant qu'azier, mèste, jusc' à la souqueto torto. » En anguent au lhèit, l'ome dissec à sa

hemno : Douman, te calera balha boun vin e boun pan au bailet. »L'endouman, la mèsto $i$ balhèc boun vin e boun pan, e atchi-le partit à la vinho.

" Adieu, vigne, vignerasse. - Qu'y a-t-il dans ta gourde? - Mauvaise piquette. - Et dans ton sac? Mauvais pain d'orge. - Couche-toi, Georges. "

Le soir, quand le valet fut de retour à la borde, le maître lui demanda : "Eh bien, valet, en as-tu fait beaucoup?

- Autant qu'hier, maître, jusqu'à la souche torte. » En allant au lit, l'homme dit à sa femme :

"Demain, il te faudra donner au valet bon vin et bon pain."

Le lendemain, la maîtresse lui donna bon vin et bon pain, et le voilà parti pour la vigne.

« Adiu, vinho, vinharasso. - Que i a dins ta coujarasso ? - Boun vin. - E dins toun sacas ? - Boun pan de blat. - Trabalho, goujat. »Le se, quand le bailet arribèc à la bordo, le mèste i demandèc : « È be, bailet, n'as pla hèit, auèi ? - Mèste, è acabat. »

«Adieu, vigne, vignerasse. - Qu'y a-t-il dans ta gourde? - Bon vin. - Et dans ton sac ? - Bon pain de blé. Travaille, goujat."

Le soir, quand le valet fut de retour à la borde, le maître lui demanda : "Eh bien, valet, en as-tu fait beaucoup, aujourd'hui?

Maître,j'ai achevé. »

\section{NOTES}

1. Voir l'acharnement des pédagogues français du XIXe siècle à faire naître, dans leurs manuels de pédagogie, l'enseignement mutuel, lancé concurremment par Bell et Lancaster en GrandeBretagne vers 1810, sur le sol national dès la fin du XVIIIe... 
2. L'essentiel des manuscrits perboscquiens se trouve au Collège d'Occitanie (19, rue de la Fonderie, 31000 Toulouse) et à la Bibliothèque d'Étude et de Patrimoine (ex. "Bibliothèque municipale », rue du Périgord) de la même ville.

3. Cf. CHANET J.-F. : L'École républicaine et les petites patries, Aubier, 1996 - THIESSE A.-M. : Ils apprenaient la France, Éd. de la Maison des sciences de l'homme, 1997.

4. Cf. CHAUVIN L. : "Inconvénients de la résidence dans le pays natal ", in L'éducation de l'Instituteur - 3e année des écoles normales. Paris, A. Picard et Kaan, pp. 265 et sq.

5. Cf. ses textes essentiels Foc nou (1904) et La tradición occitana (1905).

6. Catalogne comprise alors comme en fait foi la revue éponyme catalano-occitane publiée en 1905 à Barcelone et, déjà, de 1896 à 1904, le modeste bulletin Mount-Ségur (devenu en 1900 Mont-Segur) - édités par l'instituteur audois Pr. Estieu (1860-1939) avec la collaboration de Perbosc.

7. Perbosc sera un abonné de la première heure des Cahiers de la Quinzaine - lesquels publieront en 1902 le professeur d'école normale, héraultais d'origine, Antonin Lavergne, son Jean Coste, et La Médaille, dénonciation de l'usage du "signal » scolaire contre les patoisants.

8. Journal des Instituteurs, 1-10-1886 : « Nous le répétons, ce n'estpas l'instituteur qui tuera la langue; mais, par l'œuvre lente de l'instruction, les mots patois seront remplacés l'un après l'autrepar les mots français aux quels seront adaptées la terminaison et la prononciation patoisante. » Cité in PERBOSC A. : Manifestes occitans, Cap e Cap, 1974, p. 110.

9. Auteur d'une thèse sur la Renaissance méridionale, dont la 2e partie est consacrée à La pédagogie régionaliste. Avignon, Roumanille, 1907.

10. Perbosc publiera en 1907 à Paris aux éditions Kleinbronn des Contes licencieux d'Aquitaine, ouvrage de commande. Réédition en 1984 chez GARAE, Carcassonne.

11. Terme retenu par l'auteur pour éviter « traditionaliste ».

12. Bibliothèque d'Étude et de Patrimoine de Toulouse, manuscrit 1421, feuillet 66 et suivants, cités in BRU J. : L'anneau magique (préface à A. Perbosc), Carcassonne, GARAE, 1987.

13. À restituer dans la "guerre scolaire" de l'époque. Une partie de la correspondance de 1900-1901 porte ainsi sur le caractère plus ou moins républicain, i.e. anticlérical des inspecteurs locaux, avec qui il faut composer ou ruser sur la question de la langue. Lors de la «seconde guerre des manuels » (1909-1910), Perbosc, devenu maître à La ville dieu-du-Temple (900 h), sera importuné par une mère d'élève lectrice du Pèlerin - incident qui ne pourra que le persuader un peu plus d'accepter le poste de bibliothécaire que lui offre la ville de Montauban (1912), même s'il affirme n'y rien gagner financièrement.

14. In L'Éducateur,1er février 1959 - reproduit in Cahiers pédagogiques de l'Institut d'Études Occitanes, № 11, année 1959-60,10e année.

15. En particulier par l'intermédiaire d'Hélène Cabannes, institutrice dans l'Hérault, membre du "groupe Perbosc » à l'origine de la réflexion pédagogique dans l'Institut d'Études Occitanes. Cf. CABANAS E. : «D'un ensanhament regionalista ; in Oc, 24e année, 1946-48, pp. 76-81.

16. Une synthèse de ces deux ouvrages (parus respectivement en 1914 et 1924) a été publiée (en traduction française principalement) sous le titre Récifs et Contes populaires de Gascogne - 1, réunis par sa petite-fille S. Cézerac-Perbosc, Paris, Gallimard, 1979, avec le concours de G. Passerat et N. Sabatié.

17. Cité in Manifestes, Op. cit, p. 165.

18. Peu enclin à l'enseignement des « langues régionales » comme bien des hommes politiques occitans (tel G. Doumergue ou A. de Monzie avec qui Perbosc polémiqua en 1925) si l'on en croit une rubrique publiée à l'automne 2001 dans L'Express.

19. «Ancestrale » : terme souvent utilisé par Perbosc.

20. Conte recueilli par les élèves Alphonse Miquel (1890-1909) et Jean Montaubric (né en 1896 et devenu... valet de ferme à Beaumont-de-Lomagne). Le texte publié par Perbosc correspond à ce 
que les enfants ont entendu puis écrit : il ne met pas en œuvre le travail de normalisation linguistique entrepris par Perbosc et Estieu dans la même période.

\section{RÉSUMÉS}

Devant des proclamations trop rapides, il est utile d'aller rechercher des expériences oubliées dans notre socio - histoire de l'éducation: comme celle conduite par l'instituteur Antonin Perbosc, dans le village de Comberouger (Tarn-et-Garonne) de 1900 à 1908. Ancrée dans la modernité pédagogique de l'époque (méthodes «intuitives », «actives», Tolstoï), elle annonce les entreprises de Freinet ou Cousinet, tant dans 1'«étude du milieu» que dans l'émergence de la coopération ou du travail de groupe. Non sans relation avec les convictions politiques de leur auteur (militant laïque et fédéraliste occitan).

When faced with off the cuff statements, it can turn out to be useful to being to light now forgotten experiments from the social history of our national education system, such as that conducted by Antonin Perbosc in the village of Comberouger from 1900 to 1908. While being rooted in movements which were at that time pedagogically modem, this experiment - as much by the concept of "étude du milieu" as by the emergence of the concepts of coopération and group work - also foreshadows the work later undertaken by Freinet or Cousinet.

INDEX

Mots-clés : conte, ethnographie, instituteur, occitan

Keywords : elementary school teacher, ethnography, occitan language, tale

\section{AUTEUR}

HERVÉ TERRAL

Centre d'études des rationalités et des savoirs (CERS/UMR CNRS 51-17), Université Toulouse le Mirail 